\title{
CLINICAL PHENOTYPE AND OUTCOME OF DRESS CASES IN THE SPANISH AND LATIN-AMERICAN DILI REGISTRIES
}

\author{
Mi Lucena', I Medina-Caliz', M Almarza1, C Stephens ${ }^{1}$, M Robles-Diaz ${ }^{1}$, Mc Fernandez², P Gines ${ }^{3}$, \\ F Bessone $^{4}$, N Hernandez ${ }^{5}$, Rj Andrade ${ }^{1}$
}

${ }^{I}$ UGC Aparato Digestivo y Servicio de Farmacologia Clinica. Instituto de Investigacion Biomedica de Malaga (IBIMA). Hospital Universitario Virgen de la Victoria. Universidad de Malaga, CIBERehd, Malaga, Spain, ${ }^{2}$ Complejo Hospitalario Torrecardenas, Almeria, Spain, ${ }^{3}$ Liver Unit, Hospital Clinic, CIBERehd, Barcelona, Spain, ${ }^{4}$ Hospital Provincial del Centenario, Rosario, Argentina, ${ }^{5}$ Hospital de Clinicas, Montevideo, Uruguay

Background: Severe cutaneous adverse reactions can manifest in a wide spectrum of heterogeneous clinical presentations, including drug reaction with eosinophilia and systemic symptoms (DRESS), a severe idiosyncratic drug reaction. DRESS is characterized by presence of rash, fever, eosinophilia and/or lymphopenia, and liver injury but there is no consensus for the definition of this condition. We aimed to evaluate clinical phenotype, outcome and causative agents associated with DRESS.

Methods: DRESS cases were defined according to the International Registry of Severe Cutaneous Adverse Reaction (RegiSCAR). Demographics, clinical presentation and outcome were compared between DILI cases without hypersensitivity features (non-DRESS DILI) and DRESS cases included in the Spanish ( $\mathrm{n}=35 / 920)$ and Latin-American $(\mathrm{n}=25 / 269)$ DILI registries.

Results: Sixty patients with DRESS syndrome were identified compared to 620 non-DRESS DILI cases, with a slightly higher proportion of women in the latter group (53\% vs 50\%). Mean age at onset was similar in both groups, 53 vs 49 years. Duration of treatment and time to onset were shorter in the DRESS cases (40 vs 107 and 35 vs 95 days, respectively) although the differences were not statistically significant. All DRESS cases presented rash (three with severe toxicoderma), 19 (33\%) lymphopenia and 46 (78\%) eosinophilia. Jaundice was present in 73\% of DRESS patients and $64 \%$ of non-DRESS DILI patients. Positive autoantibodies were detected in $24 \%$ of non-DRESS DILI vs $9 \%$ of DRESS patients $(p=0.021)$. Hospitalization was higher for DRESS cases ( $78 \%$ vs $51 \%, p=0.001)$. Type of liver injury differed between the groups, with cholestatic-mixed damage predominating in DRESS (58\%), and hepatocellular damage (67\%) in non-DRESS DILI, $(\mathrm{p}=0.001)$. Severity also differed, with $14 \%$ severe and no fatal cases in DRESS and $7 \%$ severe and $5 \%$ fatal cases in non-DRESS DILI ( $\mathrm{p}=0.046$ ). The most frequent causative drugs in the DRESS group were amoxicillinclavulanate ( 7 cases), carbamazepine (7), allopurinol (5) and lamotrigine (5).

Conclusion: Compared to non-DRESS DILI, DRESS cases presented a predominance of cholestatic-mixed pattern and greater severity without mortality. Amoxicillin-clavulanate stands out as a leading cause of DRESS syndrome in the Spanish DILI Registry, while antiepileptic predominates in Latin-America, similar to previously published studies. Funding:AEMPS,FEDER(PI15-01440, PI-0274/2016, PI-0285-2016).CIBERehd-ISCIII. 